\title{
A 37-year observation of mortality in Chinese chrysotile asbestos workers
}

\author{
Xiaorong Wang, ${ }^{1}$ Eiji Yano, ${ }^{2}$ Hong Oiu, ${ }^{1}$ Ignatius Yu, ${ }^{1}$ Midori N Courtice, ${ }^{1}$ L A Tse, ${ }^{1}$ \\ Sihao Lin, ${ }^{1}$ Mianzhen Wang ${ }^{3}$
}

\section{See Editorial, p 98}

${ }^{1}$ Division of Occupational and Environmental Health, School of Public Health and Primary Care, The Chinese University of Hong Kong, Hong Kong, China ${ }^{2}$ School of Public Health, Teikyo University School of Medicine, Tokyo, Japan

${ }^{3}$ Department of Occupational Health, Huaxi School of Public Health, Sichuan University, Chengdu, China

\section{Correspondence to} Professor Xiaorong Wang, School of Public Health and Primary Care, The Chinese University of Hong Kong, 4/F School of Public Health, Prince of Wales Hospital, Shatin, N.T., Hong Kong SAR, China; xrwang@cuhk.edu.hk

Received 14 March 2011 Accepted 20 July 2011 Published Online First 21 September 2011

\begin{abstract}
Objectives This 37 -year prospective cohort study was undertaken to provide additional evidence for mortality risks associated with exposure to chrysotile asbestos.

Methods 577 asbestos workers and 435 control workers in original cohorts were followed from 1972 to 2008 , achieving a follow-up rate of $99 \%$ and $73 \%$, respectively. Morality rates were determined based on person-years of observation. Cox proportional hazard models were constructed to estimate HRs of causespecific mortality, while taking into account age, smoking and asbestos exposure level.
\end{abstract}

Results There were 259 (45\%) deaths identified in the asbestos cohort, and 96 died of all cancers. Lung cancer $(n=53)$ and non-malignant respiratory diseases $(n=81)$ were major cause-specific deaths, in contrast to nine lung cancers and 11 respiratory diseases in the controls. Age and smoking-adjusted HRs for mortality by all causes and all cancers in asbestos workers were $2.05(95 \% \mathrm{Cl} 1.56$ to 2.68 ) and 1.89 (1.25 to 2.87$)$, respectively. The risks for lung cancer and respiratory disease deaths in asbestos workers were over threefold that in the controls (HR 3.31 (95\% Cl 1.60 to 6.87); HR 3.23 (95\% Cl 1.68 to 6.22 ). respectively). There was a clear exposure-response trend with asbestos exposure level and lung cancer mortality in both smokers and non-smokers.

Conclusion Data from this prospective cohort provide strong evidence for increased mortality risks, particularly from lung cancer and non-malignant respiratory diseases, associated with exposure to chrysotile asbestos, while taking into account of the smoking effect.

Asbestos, a recognised hazard in both occupational and environmental settings, has been responsible for millions of deaths worldwide. ${ }^{1}$ Because of enormous adverse health impacts on exposed workers and the general population, production and use of all types of asbestos have been banned or restricted in 52 countries to date. ${ }^{2}$ However, chrysotile asbestos continues to be mined, manufactured and used in many other countries, with about 125 million people exposed occupationally worldwide.

The carcinogenic potency of chrysotile has long been debated. It has been claimed that only amphiboles but not chrysotile is responsible for lung cancer and mesothelioma in exposed workers, the basis of the so called 'amphibole hypothesis'. Growing evidence, however, has shown that exposure to chrysotile asbestos increases lung cancer risk, ${ }^{4-7}$ although controversy remains regarding its association with mesothelioma. ${ }^{8}$

\section{Key messages}

What is the key question?

- What is the major cause-specific mortality in the cohort of workers who were exposed to chrysotile asbestos, and how strong are the associations between the cause-specific mortality and asbestos exposure?

\section{What is the bottom line?}

- This 37-year prospective cohort study observed significantly greater mortality of all causes, and all cancers in the asbestos workers, in comparison with the controls. However, the strongest association with asbestos exposure was seen in lung cancer and non-malignant respiratory disease, which showed a clear exposure-response trend.

\section{Why read on?}

- Chrysotile asbestos continues to be mined and used heavily in China, largely because a controversy remains on carcinogenic potency of chrysotile asbestos. This prospective cohort study with the longest observation time to date, and high follow-up rate delivers a strong message that exposure to chrysotile asbestos can cause substantially high mortality risk for lung cancer as well as non-malignant respiratory disease.

China is one of the biggest asbestos producers and users in the world, with total asbestos production increasing yearly. ${ }^{9}$ There were an estimated 100000 workers employed in asbestos mines and asbestos product factories, with an unknown number working in small mines and factories. ${ }^{10}$ In 2001, a 25-year follow-up study in an asbestos textile factory reported excessive lung cancer mortality. ${ }^{11}$ However, that study only observed $25.6 \%$ deaths in the cohort and did not report on other cause-specific mortalities. More deaths and lung cancer cases were expected during the following decade, as the workers aged. In the present study, we continued observation up to 37 years and analysed cause-specific mortality, including non-malignant respiratory diseases and gastrointestinal cancer. The primary objective was to obtain more convincing evidence for the association between exposure to chrysotile asbestos and the risks of cancers and non-malignant lung diseases. The results from the study are of interest 
for both the scientific community and policy makers in the countries where production or use of asbestos is yet to be banned or restricted.

\section{SUBJECTS AND METHODS Study setting}

The cohort study was undertaken in an asbestos manufacturing factory in China. Detailed information on the factory was described previously. ${ }^{11}$ Periodic total dust measurements from different workshops showed that concentrations were very high. Air samples were taken in 1999 from the different workshops for measurement of both fibre and dust concentrations. Fibre concentrations were high in raw material and textile sections, and low in asbestos rubber and cement sections. ${ }^{11}$ Both area and personal samples were taken in 2002 (table 1), which indicated a similar trend. Analysis of available chrysotile samples by $\mathrm{X}$-ray diffraction and transmission electron microscopy analyses indicated a very low level of tremolite fibre. ${ }^{11}$

\section{Cohorts and follow-up}

The original cohort was established with 586 male asbestos workers who were on the factory roster as of 1 January 1972. There were seven major job categories, ${ }^{11}$ and job tasks held by most of the workers were stable. At the same time, 599 male workers without exposure to asbestos or other industrial dusts were recruited as a control cohort from an electronics equipment factory located in the same city. The scale of the factory was comparable to the asbestos factory, and the included workers accounted for about $95 \%$ of male workers in the factory. The cohorts were followed prospectively through 31 December 2008, and vital status information was obtained using a combination of active follow-up and record linkages to death certificates kept in the factories and the municipal death registry. Personnel records including workers' addresses and vital status information were well maintained in the asbestos factories, which made the cohort members relatively traceable. By the end of 2008, we successfully followed 577 asbestos workers from the original cohort and lost only 9 workers, achieving a 99\% follow-up rate over 37 years. In the control cohort, many workers had relocated to other cities or provinces after retiring over the last decade, making follow-up more challenging. In spite of that, we traced 435 workers (73\%). Detailed information was obtained by personal interviews. A structured questionnaire was used to collect individual data mainly during the period of 1997 and 2008, based on the last follow-up conducted in the end of 1996. The collected information included workers' jobs held in the asbestos factory, job title changes and duration of each job title held, employment status and retirement date since last follow-up. The information on workers' working history was verified from factory personnel records, especially for those who died or were not able to recall clearly. In addition, smoking information was collected. For those who had died, the information was obtained from worker's spouse/next of kin. For those deceased, the date and underlying cause of death were retrieved from hospitals and a death registry.

Table 1 Fibre concentrations $\left(\mathrm{f} / \mathrm{cm}^{3}\right)$ of area and personal samples by workshop (2002)

\begin{tabular}{lrlrrl}
\hline & \multicolumn{2}{l}{ Area samples } & & \multicolumn{2}{l}{ Personal samples } \\
\cline { 2 - 3 } & $\mathbf{N}$ & Median $\left(\mathbf{P}_{\mathbf{2 5}}-\mathbf{P}_{\mathbf{7 5}}\right)$ & & $\mathbf{N}$ & Median $\left(\mathbf{P}_{\mathbf{2 5}}-\mathbf{P}_{\mathbf{7 5}}\right)$ \\
\hline Raw material & 31 & $7.2(1.0-18.3)$ & & $8.5(1.8-9.4)$ \\
Textile & 62 & $2.1(0.6-10.0)$ & & 23 & $8.6(5.7-9.8)$ \\
Rubber plate & 5 & $0.8(0.7-1.1)$ & & 1 & 1.5
\end{tabular}

Few samples from the rubber plant and no samples from the cement section were taken in 2002 because of too few workers working in the sections at that time.
Information on diagnostic means of malignant tumours was gathered. The study was approved by the Research Ethics Committee of the Chinese University of Hong Kong.

\section{Statistical analysis}

Primary outcomes of the study were cause-specific mortality including lung cancer, gastrointestinal cancer and non-malignant respiratory diseases over 37 years. Data analysis focused on a comparison of these outcome variables between the asbestos workers and the controls. Workers were classified as either nonsmokers or ever-smokers because specific smoking amount was either missing or uncertain in some workers. The latter were defined as having smoked at least one cigarette per day for 6 months or longer. Person-years of observation were accrued from the date of entry into the cohorts until the date of death or the end of follow-up among those who were followed in this study. Age-adjusted mortality rates of different causes were calculated based on the national male population age distribution in corresponding time periods. ${ }^{12}$ Kaplan-Meier curves were plotted to compare cumulative survival of lung cancer and all cancers between the two cohorts. The difference was tested with a log-rank test. Cox proportional hazard models were applied to evaluate the effects of asbestos exposure, smoking status and age on lung cancer and other cause-specific mortalities, using length of follow-up as the time dimension. Two different models were constructed: in the first model, exposure status, that is, asbestos workers against the controls, was included. In the second model, asbestos workers were grouped into three exposure levels based on their job types: high level (those in raw material and textile sections), medium level (maintenance workers who were responsible for maintaining and repairing machines in all workshops) and low level (those in rubber, cement, administration and other services). We created three dummy variables with control workers as the reference to determine a possible exposur$\mathrm{e}-$ response relationship with exposure levels. Finally, we assessed the joint effect of exposure to asbestos and smoking on lung cancer mortality in the two cohorts, in which non-smoking control workers served as the reference group. We calculated a synergy index to test the interaction departure from an additive model and used a bootstrapping re-sampling method to calculate 95\% CI. The data analysis was carried out with the Statistical Package for the Social Sciences Software V.16.0 for Windows.

\section{RESULTS}

The observational period generated 17508 and 15013 personyears in asbestos workers and the controls, respectively. Table 2 provides basic demographics, in which average age at entry was a little higher in asbestos workers than in the controls (40 vs 35 years old). Smoking was more common in asbestos workers than in the controls (78\% vs $54 \%$ ). In asbestos workers, average age at start of asbestos exposure was 29 years, and average total duration of exposure was 25 years. There were 127 asbestos workers diagnosed with asbestosis, accounting for $22 \%$ in the cohort.

There were 259 deaths (45\%) in the asbestos workers, in contrast to $76(18 \%)$ in the controls (table 2). Ninety-six asbestos workers died from all cancers, accounting for $37 \%$ of all deaths, of which 53 died from lung cancer, 29 from gastrointestinal (GI) cancers and 2 from mesothelioma. An additional 81 workers $(31.3 \%)$ died from non-malignant respiratory diseases, among whom, there were 39 workers diagnosed with asbestosis In the control cohort, 33 (43\%) workers died from all cancers, including nine lung cancers and 18 GI cancers. An additional 11 $(14 \%)$ died from non-malignant respiratory diseases. 
Table 2 Basic demographic data* and identified vital status in asbestos and control cohorts over 37 years

\begin{tabular}{|c|c|c|}
\hline & $\begin{array}{l}\text { Asbestos } \\
\text { workers }(n=577)\end{array}$ & $\begin{array}{l}\text { Control workers } \\
(n=435)\end{array}$ \\
\hline Total person-years of follow-up & 17507.83 & 15013.25 \\
\hline Year of birth (median (P25, P75)) & $1930(1922,1944)$ & $1938(1932,1944)$ \\
\hline Age at entry (years) & $39.7(11.8)$ & $34.8(8.3)$ \\
\hline Age at end of follow-up (years) & $70.1(11.1)$ & $69.3(8.9)$ \\
\hline Age at death for decedents (years) & $68.2(11.2)$ & $64.8(14.3)$ \\
\hline Age at exposure start & $29.0(8.5)$ & - \\
\hline Years of exposure at entry & $10.8(6.7)$ & - \\
\hline Total years of exposure & $25.3(7.8)$ & - \\
\hline Smoking (n, \%) & $452(78.3)$ & $236(54.3)$ \\
\hline Asbestosis (n, \%) & $127(22.2)$ & \\
\hline \multicolumn{3}{|l|}{ Identified deaths } \\
\hline All causes & $259(44.9)$ & $76(17.5)$ \\
\hline All cancers $\dagger$ & $96(37.1)$ & $33(42.9)$ \\
\hline Lung cancer & $53(20.5)$ & $9(11.7)$ \\
\hline Gl cancer $\ddagger$ & $29(11.2)$ & $18(23.4)$ \\
\hline Mesothelioma & $2(0.8)$ & $0(0.0)$ \\
\hline NMRD§ & $81(31.3)$ & $11(14.3)$ \\
\hline
\end{tabular}

*Values denote mean (SD), unless otherwise specified.

tDeaths of specific causes were calculated among all deaths.

‡Gastrointestinal cancers including stomach (gastric), liver, oesophageal, colorectal cancers and bile duct cancer.

$\S N o n-m a l i g n a n t$ respiratory diseases.

Age-adjusted cause-specific mortality rates were considerably higher in asbestos workers (table 3), particularly for lung cancer and non-malignant respiratory diseases, with 9.6-fold and 6.4-fold that in the controls, respectively. GI cancer mortality in asbestos workers was not higher than in the controls. Asbestos workers had significantly shorter cumulative survival for all cancers and lung cancer $(p<0.001)$ or greater cumulative mortality over the observation time (figure 1).

In multivariate analysis (table 4), exposure to asbestos was significantly associated with greater risks for all cause-specific deaths, except for GI cancer (model 1). Relative to the controls, there was a twofold death risk for all causes and all cancers, and a threefold risk for lung cancer and non-malignant respiratory diseases in asbestos workers. Asbestos workers were further classified into three exposure levels, in which 171 workers $(30 \%)$ were in the high, 290 workers (50\%) in the medium and 116 workers $(20 \%)$ in the low exposure level. In the models that included the three exposure levels (model 2), even those at the low exposure level had excessive risks for all cause-specific deaths compared to the controls. The risks tended to be greater with higher exposure level, the clearest gradient being seen for lung cancer and non-malignant respiratory diseases. Those at the high exposure level had a sixfold risk for lung cancer death (adjusted HR 6.01, 95\% CI 2.74 to 13.19). Smoking was a significant contributing factor to the increased risk for lung cancer mortality in either model. Age was significantly associated with all cause-specific deaths.

Table 3 Age-adjusted mortality rates (deaths $/ 10^{5}$ person-years) and $95 \% \mathrm{Cl}$

\begin{tabular}{lll}
\hline & Asbestos workers $(\mathbf{n}=\mathbf{5 7 7})$ & Control workers $(\mathbf{n}=\mathbf{4 3 5})$ \\
\hline All causes & $1560.36(1375.32$ to 1745.39$)$ & $544.28(426.26$ to 662.29$)$ \\
All cancers & $714.61(589.39$ to 839.83$)$ & $235.25(157.66$ to 312.84$)$ \\
Lung cancer & $588.50(474.87$ to 702.14$)$ & $61.23(21.65$ to 100.82$)$ \\
Gl cancer* & $76.45(35.49$ to 117.41$)$ & $153.10(90.51$ to 215.69$)$ \\
NMRD† & $549.09(439.32$ to 658.85$)$ & $85.73(38.90$ to 132.57$)$ \\
\hline
\end{tabular}

*Gastrointestinal cancers.

†Non-malignant respiratory diseases.
Joint effect analysis adjusting for age at entry showed that both smoking and asbestos exposure, separately, increased the risk of lung cancer mortality (table 5). The greatest risk, however, was observed in both smoking and asbestos exposure combined (adjusted HR 17.35; 95\% CI 2.38 to 126.57). The synergy index was 1.414 (95\% CI 0.734 to 3.987 ), indicating that the joint effect was more than additive, though not statistically significant. When stratified by exposure levels, asbestos workers who smoked had a greater risk than their non-smoking counterparts at the low and medium exposure levels, but not at the high level. Risk of lung cancer clearly increased with exposure level in both smokers and non-smokers. Smokers in all three exposure levels had a significantly greater risk in comparison with the reference group. The highest risk was observed in smokers at the high asbestos exposure level, followed by nonsmokers at the high asbestos exposure level, both of which were statistically significant despite wide CIs.

\section{DISCUSSION}

Exposure to asbestos will continue to be a public health challenge in many countries, despite increased efforts to ban asbestos internationally. According to updated information from the WHO, more than 107000 people die each year from asbestos-related lung cancer, mesothelioma and asbestosis, and one in every three occupational cancer deaths is attributable to asbestos. ${ }^{1}$ In this study, we prospectively followed a heavily exposed group of asbestos workers, with average 25 years of exposure, for 37 years. The concentrations of either total dust or fibres far exceeded the already lenient national standards (2 mg/ $\mathrm{m}^{3}$ before 2001, and $0.8 \mathrm{mg} / \mathrm{m}^{3}$ and $0.8 \mathrm{f} / \mathrm{ml}$ since 2002). According to factory records, only chrysotile was ever used in the factory. Although not all samples of asbestos historically used were available, the analyses of available samples have indicated that the contamination of amphibole was very low. ${ }^{11}$ Since the 25-year study, the number of deaths in both cohorts had nearly doubled, which enhanced the statistical power of the study. In addition, we analysed mortality from gastrointestinal cancer and non-malignant respiratory diseases in the cohort, which were not reported previously.

We observed 53 lung cancer deaths, accounting for a fifth of all deaths in the cohort. An additional 81 (31\%) workers died of non-malignant respiratory diseases, including asbestosis and related complications. Age-adjusted mortality from lung cancer and respiratory diseases in the asbestos workers was as high as 9.6 and 6.4 times that in the controls, respectively, suggesting that asbestos-related lung cancer and respiratory diseases were major causes of deaths in these workers. The results were corroborated in the multivariate analyses, showing the greatest risks associated with the exposure for lung cancer and nonmalignant respiratory diseases. The estimated risk for either mortality outcome was over three times that in the controls. In comparison with the controls, even those at the low exposure level had a twofold risk for lung cancer death and a 2.5-fold risk for death from respiratory diseases. Moreover, the analyses showed a clear exposure-response trend, with a sixfold risk of lung cancer death at the high exposure level. A similar gradient was also seen in non-malignant respiratory disease deaths, with a nearly fivefold risk for those at the high exposure level. These results suggest strong associations between asbestos exposure and lung cancer and non-malignant respiratory diseases.

The carcinogenicity of asbestos has been well recognised for over half a century. However, the association between exposure to chrysotile asbestos and lung cancer remains a topic of interest, as this type has been, and continues to be, widely used 
Figure 1 Kaplan-Meier curves for cumulative survival from all cancers and lung cancer in asbestos workers and controls.

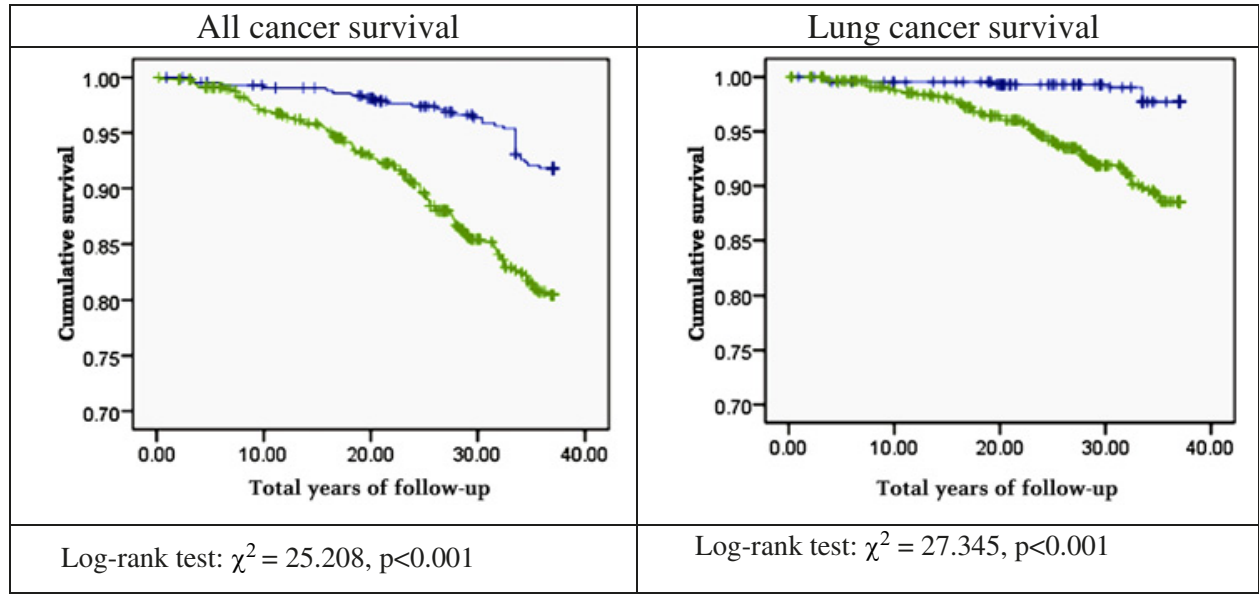

Asbestos cohort

\section{- Control cohort}

in many countries. In addition, the debate about the relative carcinogenicity of chrysotile and amphiboles is ongoing. Existing data from cohort studies conducted in the asbestos textile industry, though limited in number, have provided evidence for a positive association between chrysotile and lung cancer. There is an increasing consensus that there is virtually no difference between lung cancer risks presented by the different fibre types. ${ }^{7} 8$ Retrospective cohort studies in South Carolina textile workers observed SMR for lung cancer that was twofold that of the general population with an exposure-response relationship. ${ }^{7} 13$ A recent retrospective cohort study in North Carolina observing workers employed for at least 1 day in any of four asbestos textile plants also reported a nearly doubled SMR for lung cancer and an increased risk with cumulative fibre exposure. ${ }^{7}$ Another retrospective cohort study conducted in Italian workers employed for at least 1 month in an asbestos textile company showed a similar finding of increased lung cancer mortality. ${ }^{14}$ On the other hand, most of the previous studies were limited by their retrospective design, particularly the use of historical data and a lack of smoking information in study subjects.

As seen in other countries, smoking was common in asbestosexposed workers in China. This was reflected in our asbestos worker cohort with $78 \%$ smokers, compared with $54 \%$ in the control cohort. Smoking was found to be a significant contributor to lung cancer in the multivariate analysis. However, a further analysis of lung cancer showed that even non-smoking asbestos workers had a greater risk than the controls. Further- more, a clear exposure-response gradient was seen not only in the smokers but also in the non-smokers. These imply that smoking by no means was the only explanation for the difference in lung cancer mortality between the two cohorts. An interactive effect between smoking and asbestos exposure on lung cancer was demonstrated; a greater risk was observed in asbestos workers who also smoked (HR 17.4) than those who did not smoke (HR 7.5). The synergy index was 1.41, indicating more than an additive effect of both factors. This result was consistent with other findings. ${ }^{13} 15-17$ The absence of statistical significance could be explained by inadequate study power due to a limited number of lung cancer cases. In a stratified analysis of asbestos workers by three exposure levels, the interactive effect was seen at the low and medium exposure levels. However, there was little evidence of an interactive effect shown at the high exposure level, in which the smokers and their non-smoking counterparts appeared to have the same size of risk. This phenomenon showing different effects with different exposure levels might reflect an unstable result due to a small sample size resulting from the stratification. Alternatively, it might reflect reality, as the high levels of asbestos these workers were exposed to have rarely been seen in western countries and previous studies addressing the asbestos-smoking interaction. ${ }^{13} 17$ We were not able to draw a conclusion from the current data, but it may be worthwhile to study the phenomenon further with a larger sample data.

Two cases of mesothelioma, one pleural and one peritoneal, were observed in the cohort, and another case was reported in

Table 4 HRs $(95 \% \mathrm{Cl})$ for death due to all causes and cancer causes in asbestos workers and controls

\begin{tabular}{|c|c|c|c|c|c|}
\hline & All causes $(n=335)$ & All cancers $(n=129)$ & Lung cancer $(n=62)$ & GI cancer* $(n=47)$ & NMRD $†(n=92)$ \\
\hline \multicolumn{6}{|l|}{ Model $1 \neq$} \\
\hline Asbestos exposure & 2.05 (1.56 to 2.68$)$ & $1.89(1.25$ to 2.87$)$ & $3.31(1.60$ to 6.87$)$ & $1.14(0.61$ to 2.14$)$ & 3.23 (1.68 to 6.22$)$ \\
\hline Smoking & $1.01(0.79$ to 1.30$)$ & $1.18(0.79$ to 1.78$)$ & $2.78(1.26$ to 6.15$)$ & 0.85 (0.46 to 1.58$)$ & 1.25 (0.75 to 2.08$)$ \\
\hline Age at entry & 1.09 (1.07 to 1.10$)$ & 1.07 (1.05 to 1.09$)$ & $1.07(1.05$ to 1.10$)$ & $1.06(1.03$ to 1.10$)$ & $1.13(1.11$ to 1.16$)$ \\
\hline \multicolumn{6}{|l|}{ Model 2§ } \\
\hline Low level & 1.79 (1.33 to 2.42$)$ & 1.57 (0.98 to 2.52$)$ & $1.94(0.84$ to 4.46$)$ & $1.39(0.70$ to 2.76$)$ & 2.55 (1.27 to 5.13$)$ \\
\hline Medium level & 1.93 (1.32 to 2.82 ) & 1.61 (0.87 to 2.98$)$ & 3.49 (1.41 to 8.67$)$ & 0.83 (0.28 to 2.49$)$ & 3.50 (1.56 to 7.84$)$ \\
\hline High level & 2.72 (1.98 to 3.75$)$ & 2.83 (1.73 to 4.62$)$ & 6.01 (2.74 to 13.19$)$ & 0.87 (0.33 to 2.29$)$ & 4.76 (2.31 to 9.77$)$ \\
\hline $\mathrm{p}$ for trend $\boldsymbol{\Phi}$ & $<0.001$ & $<0.001$ & $<0.001$ & 0.677 & $<0.001$ \\
\hline Smoking & $0.98(0.76$ to 1.25$)$ & $1.13(0.75$ to 1.71$)$ & $2.54(1.14$ to 5.63$)$ & 0.87 (0.47 to 1.61$)$ & $1.13(0.67$ to 1.91$)$ \\
\hline Age at entry & 1.09 (1.07 to 1.10$)$ & 1.07 (1.05 to 1.09$)$ & $1.07(1.04$ to 1.10$)$ & $1.06(1.03$ to 1.09$)$ & $1.13(1.11$ to 1.16$)$ \\
\hline
\end{tabular}

*Gastrointestinal cancers.

†Non-malignant respiratory diseases.

$\neq$ Control workers served as reference group.

$\S$ Asbestos workers at each exposure level were compared with the control workers.

TTrend test for the three exposure categories. 
Table 5 Joint effect (HR and $95 \% \mathrm{Cl}$ ) of exposure to asbestos and smoking on lung cancer mortality in asbestos workers and controls

\begin{tabular}{lll}
\hline & Non-smoking & Smoking \\
\hline Control cohort & 1.00 & 6.03 (0.75 to 48.21$)$ \\
Asbestos cohort & $7.52(0.90$ to 62.79$)$ & 17.35 (2.38 to 126.57$)$ \\
$\quad$ Synergy index & $1.4141(0.734$ to 3.987$)$ & \\
$\quad$ Low level $\dagger$ & $2.06(0.13$ to 33.17$)$ & $10.71(1.41$ to 81.58$)$ \\
$\quad$ Medium level & $6.39(0.40$ to 102.17$)$ & $18.43(2.33$ to 145.88$)$ \\
$\quad$ High level & $26.15(2.91$ to 234.88$)$ & $28.58(3.82$ to 213.64$)$ \\
\hline
\end{tabular}

${ }^{*} \mathrm{HR}$ and $95 \% \mathrm{Cl}$ using Cox model, adjusting for age at entry.

†Non-smoking controls served as reference group in comparison with asbestos workers in different exposure levels.

a family member who spun chrysotile asbestos at home during childhood. ${ }^{18}$ One possible reason for the small number of mesothelioma cases in the cohort might be related to the type of asbestos the workers were exposed to. Systematic reviews have suggested that fibre types may differ in their potency for causing mesothelioma, though the result for lung cancer is less clear. ${ }^{8} 15$ In addition, we did not observe an excessive risk for gastrointestinal cancer mortality in the cohort. Some studies have found slightly elevated gastrointestinal cancer deaths in asbestos workers, ${ }^{14}$ but others have not. ${ }^{719}$ The inconsistent results in different studies may imply that asbestos is not strongly associated with gastrointestinal cancer. There may be some alternative explanations for the higher risk in certain studied populations.

This study has important strengths, including a long followup time and a high follow-up rate, with $99 \%$ in the asbestos cohort. Even in the control cohort, the rate of $73 \%$ was reasonably high for such a long observation time. To the best of our knowledge, this is one of few cohort studies that have observed asbestos workers prospectively for nearly four decades. Moreover, information on individual smoking was available, which was rarely achieved in previous studies. Unlike many other studies that used the general population as a reference, the present study utilised an industrial control cohort without exposure to known industrial carcinogens, which helps to overcome the 'healthy or unhealthy worker effect' resulting from comparisons with a general population.

There are several limitations to point out. First, asbestos dust concentrations were measured periodically in the different workshops, but fibre concentrations and personal samples were not available until 1999. The lack of historical fibre data prevented us from characterising exposure more reliably for these workers. We defined three exposure categories as surrogates of workers' exposure level and detected a clear exposureresponse trend, which suggested that the surrogate exposure definitions reflected the workers' true exposure levels to a large extent. However, misclassification of exposure remained likely, which could have led to an underestimation of the associations under study. Second, about half of the malignant tumours in the cohorts were confirmed pathologically, either by biopsy or autopsy. The others were diagnosed on the basis of CT and clinical course. Yet, the misdiagnosis or misclassification, if any, would be present randomly in the two cohorts, as the diagnoses were made at municipal level hospitals, rather than by any of the investigators. Thus, it would not change the direction of the associations and the size of risk estimation substantially. Finally, despite the high follow-up rate in the asbestos worker cohort, there were $27 \%$ of the control workers who were lost to followup. If a dissimilar proportion of deaths and causes of death occurred in those lost to follow-up, a concern over selection bias may have arisen due to differential loss to follow-up. Although loss to follow-up in these workers was mainly related to moving out from the city, the possibility of a difference in vital status between those followed and those lost could not be ruled out. Thus, the loss to follow-up in the control cohort could have led to an overestimation of effect estimates in this study, if a larger number in the lost died from lung cancer and other causes of interest.

In summary, the data from this 37-year prospective cohort confirmed excessive risks for mortality, in particular, from lung cancer and non-malignant respiratory diseases, in asbestos workers. The increased cause-specific mortality was most likely attributable to chrysotile asbestos exposure, given that the effect of smoking was considered and the increased risk was also observed in non-smokers.

Acknowledgements The authors wish to thank Ms Du Lili and Mr Mou Jinfeng for their participation in data collection and all workers in the two factories for their cooperation.

Funding This study was funded by the Pneumoconiosis Compensation Funding Board, Hong Kong SAR, China.

\section{Competing interests None declared.}

Ethics approval Ethics approval was provided by the Research Ethics Committee of the Chinese University of Hong Ko.

Contributors We assure that all authors included on a paper fulfil the criteria of authorship. There is no one else who fulfils the criteria who has not been included as an author. Professors Wang XR, Yano E, Yu I and Wang MZ worked on the conception and design of the study. Professor Wang XR drafted the manuscript. Dr Qiu H, Lin SH and Tse T worked on data analysis and interpretation and presentation of results. Ms Courtice M participated in critical revision and edition of the manuscript. All authors gave final approval of the revision.

Provenance and peer review Not commissioned; externally peer reviewed.

\section{REFERENCES}

1. World Health Organization. Asbestos: Elimination of Asbestos-Related Diseases, 2010. http://www.who.int/mediacentre/factsheets/fs343/en/index.html.

2. International Ban Asbestos Secretariat. Current Asbestos Bans and Restrictions. Compiled by Laurie Kazan-Allen. http://ibasecretariat.org/alpha_ban_list.php (accessed 8 Dec 2010).

3. Mossman BT, Bignon J, Corn M, et al. Asbestos: scientific developments and implications for public policy. Science 1990;247:294-301.

4. Stayner LT, Dankovic DA, Lemen RA. Occupational exposure to chrysotile asbestos and cancer risk: a review of the amphibole hypothesis. Am J Public Health 1996;86:179-86.

5. Cullen MR. Chrysotile asbestos: enough is enough. Lancet 1998;351:1377-8.

6. Hein MJ, Stayner LT, Leyman E, et al. Follow-up study of chrysotile textile workers: cohort mortality and exposure-response. Occup Environ Med 2007;64:616-25.

7. Loomis D, Dement JM, Wolf SH, et al. Lung cancer mortality and fibre exposures among North Carolina asbestos textile workers. Occup Environ Med 2009;66:535-42.

8. Berman DW, Crump KS. A meta-analysis of asbestos-related cancer risk that addresses fiber size and mineral type. Crit Rev Toxicol 2008;38(Suppl 1):49-73.

9. China Asbestos Association. http://www.smxh.org/ReadNews.asp?Newsld=267 (accessed 10 Sep 2010).

10. Feng Y, Liu J, Zhang T, et al. Asbestos in China: country report. Proceedings of the Asbestos Symposium for Asian Countries. Finnish Institute of Occupational Health, 2002.

11. Yano E, Wang ZM, Wang XR, et al. Longitudinal study of the mortality of lung cancer in chrysolite workers. Am J Epidemiol 2001;54:538-43.

12. National Statistical Bureau of China. China Population Statistics Yearbook: 1980-2000. http://www.docin.com/p-80012164.html.

13. Stayner L, Smith R, Bailer J, et al. Exposure-response analysis of risk of respiratory disease associated with occupational exposure to chrysotile asbestos. Occup Environ Med 1997:54:646-65.

14. Pira E, Pelucchi C, Buffoni L, et al. Cancer mortality in a cohort of asbestos textile workers. Br J Cancer 2005;92:580-6.

15. Hodgson JT, Darnton A. The quantitative risks of mesothelioma and lung cancer in relation to asbestos exposure. Ann Occup Hyg 2000;44:565-601.

16. Rothman KJ. Synergy and antagonism in cause-effect relationships. Am J Epidemiol 1976;103:506-11.

17. Lee PN. Relation between exposure to asbestos and smoking jointly and the risk of lung cancer. Occup Envrion Med 2001;58:145-53.

18. Yano E, Wang ZM, Wang XR, et al. Mesothelioma in a worker who spun chrysotile asbestos at home during childhood. Am J Ind Med 2009;52:282-7.

19. Berry G. Mortality and cancer incidence of workers exposed to chrysotile asbestos in the friction-products industry. Ann Occup Hyg 1994;38:539-46. 